\title{
Effect of New ERA Coagulant on Paper Mill Waste Water
}

\author{
Dr. Arif Khan \\ Principal, M-Tech, \\ Nuva College of Engineering and Technology, \\ Nagpur, India.
}

\author{
Mr. Ritesh R. Tandekar \\ Principal, M-Tech, \\ Nuva College of Engineering and Technology, \\ Nagpur, India.
}

\begin{abstract}
The waste water discharge from industry possess a potent threat to the existence of aquatic wildlife, mainly due to presence of high value of COD, TSS, Acidity and Alkalinity. The treatment of such waste water is today's inevitable necessity. Therefore, a more convenient way of treatment considering economic constraints needs to be suggested. This paper delves into the realm of exploiting the impact of new era coagulants on the properties of identified industrial effluent. This paper deals with the review of comparative study of performance of new era coagulants viz. poly aluminium chloride (PAC), aluminium chlorohydrate (ACH), magnesium chloride and poly-glu which can contribute to make primary treatment in a more comprehensive manner.
\end{abstract}

Keywords-Paper mill waste water, Aluminium Chloride (PAC) Aluminium Chlorohydrate (ACH) Poly-glu.

\section{INTRODUCTION}

Now a days, it is very important to treat the industrial waste waters on a serious note. The large amount of growth in industrial waste waters is to be observed with respect to increasing number of industries. The direct discharge of such industrial waste water into the environment sources create harmful effects to humans, animals and plants. The self-purification capability of the industrial waste waters is less in order to neglect the various pollution problem. The two major sources of adulterated waste water are industrial and domestic waste. Domestic sewage carries approximate $70 \%$ of waste water and remaining is carried by industries and other. More effective method is required to treat industrial waste water. In these paper, used new era coagulant for the treatment of paper mill waste water like
1. Aluminum Chlorohydrate $(\mathrm{ACH})$
2. Magnesium Chloride $(\mathrm{MgCl} 2.6 \mathrm{H} 2 \mathrm{O})$
3. Poly Aluminum Chloride (PAC) and
4. Poly-Glu

\section{LITRATURE REVIEW}

\begin{tabular}{|c|c|c|c|}
\hline $\begin{array}{l}\text { Sr. } \\
\text { No }\end{array}$ & Name of Author \& Journal & Title Of Paper & Conclusion \\
\hline 1 & $\begin{array}{l}\text { A.P. Baksh, } \\
\text { A.M. Mokadam } \\
\text { International Research Journal of } \\
\text { Engineering and Technology (IRJET) } \\
\text { Feb2019 }\end{array}$ & $\begin{array}{l}\text { Effects of New Era } \\
\text { Coagulants on Properties of } \\
\text { Industrial Wastewater: An } \\
\text { Overview }\end{array}$ & $\begin{array}{l}80 \% \text { Effect of } \mathrm{pH} \text { on } \% \mathrm{COD} \text { removal for different coagulants } \\
(\mathrm{CuSO} 4.5 \mathrm{H} 2 \mathrm{O}=5 \mathrm{gm} / 1, \mathrm{AlCl} 3=5 \mathrm{gm} / 1, \mathrm{PAC}=5 \mathrm{ml} / 1) \text { COD reduction) }\end{array}$ \\
\hline 2 & $\begin{array}{l}\text { Akshaya Kumar Verma, Puspendu } \\
\text { Bhunia, and Rajesh Roshan Dash } \\
\text { International Journal of Environmental } \\
\text { Science and Development, Vol. 3, No. } \\
\text { 2, April } 2012\end{array}$ & $\begin{array}{l}\text { Supremacy of Magnesium } \\
\text { Chloride for } \\
\text { Decolourisation } \\
\text { of Textile Wastewater: A } \\
\text { Comparative Study on the } \\
\text { Use } \\
\text { of Different Coagulants }\end{array}$ & $\begin{array}{l}\text { Decolourisation and COD reduction efficiency of coagulants significantly } \\
\text { depends upon the pH of wastewater. } \\
\text { Pre-hydrolysed coagulants such as } \mathrm{PACl} \text { and } \mathrm{ACH} \text { were found to be } \\
\text { effective in decolourising the wastewaters containing direct and disperse } \\
\text { dyes. Further, magnesium } \\
\text { chloride in combination with lime was found to be the best over the other } \\
\text { coagulants for decolourisation and CODreduction of textile wastewater } \\
\text { containing all the three dyes. }\end{array}$ \\
\hline 3 & $\begin{array}{l}\text { Meena Solanki, S. Suresh*, Shakti } \\
\text { Nath Das, Kanchan Shukla ICGSEE- } \\
\text { 2013[14th - 16th March 2013] } \\
\text { International Conference on Global } \\
\text { Scenario in Environment and Energy }\end{array}$ & $\begin{array}{l}\text { Treatment Of Real Textile } \\
\text { Wastewater Using } \\
\text { Coagulation Technology }\end{array}$ & $\begin{array}{l}\text { The higher charge density of poly aluminium chloride } \\
\text { Species often results in a decrease in the coagulant dose and the associated } \\
\text { solids production. These coagulants have the advantage of being more } \\
\text { effective at lower temperatures and a boarder } \mathrm{pH} \text { range than alum }\end{array}$ \\
\hline
\end{tabular}

\section{III.METHODOLOGI}

$>$ Sample collection, transportation and preservation

$>$ Pre-treatment parameters determination

$>$ Sample preparation
$>$ Treatment

$>$ Post-treatment parameters determination

$>$ Recording and analysis of results 
IV RESULT

Table 4.1: Optimum dose determination for PAC

Sample: $500 \mathrm{ml}, \mathrm{pH}: 4$

\begin{tabular}{|c|c|c|c|c|c|c|c|c|c|}
\hline \multirow[b]{2}{*}{$\begin{array}{c}\text { Dose } \\
(\mathrm{mg} / \mathrm{L})\end{array}$} & \multicolumn{3}{|c|}{ Initial } & \multicolumn{3}{|c|}{ Final } & \multicolumn{3}{|c|}{ Percent reduction } \\
\hline & $\begin{array}{c}\text { COD } \\
(\mathrm{mg} / \mathrm{L})\end{array}$ & $\begin{array}{c}\text { TSS } \\
(\mathrm{mg} / \mathrm{L})\end{array}$ & $\begin{array}{c}\text { Turbidity } \\
\text { (NTU) }\end{array}$ & $\begin{array}{c}\mathrm{COD} \\
(\mathrm{mg} / \mathrm{L})\end{array}$ & $\begin{array}{c}\text { TSS } \\
(\mathrm{mg} / \mathrm{L})\end{array}$ & $\begin{array}{c}\text { Turbidity } \\
\text { (NTU) }\end{array}$ & COD & TSS & Turbidity \\
\hline 500 & 925 & 990 & 670 & 435 & 105 & 82.7 & 54.4 & 90.3 & 86.5 \\
\hline 1000 & 925 & 990 & 670 & 440 & 71 & 65.9 & 41.2 & 91.35 & 89.56 \\
\hline 1500 & 925 & 990 & 670 & 415 & 82 & 71 & 50.12 & 91.02 & 87.5 \\
\hline 2000 & 925 & 990 & 670 & 395 & 105 & 86 & 54.3 & 90.6 & 85.2 \\
\hline 2500 & 925 & 990 & 670 & 385 & 125 & 94.6 & 55.4 & 88.84 & 83.5 \\
\hline 3000 & 925 & 990 & 670 & 370 & 375 & 124.5 & 58.4 & 87.5 & 81.41 \\
\hline
\end{tabular}

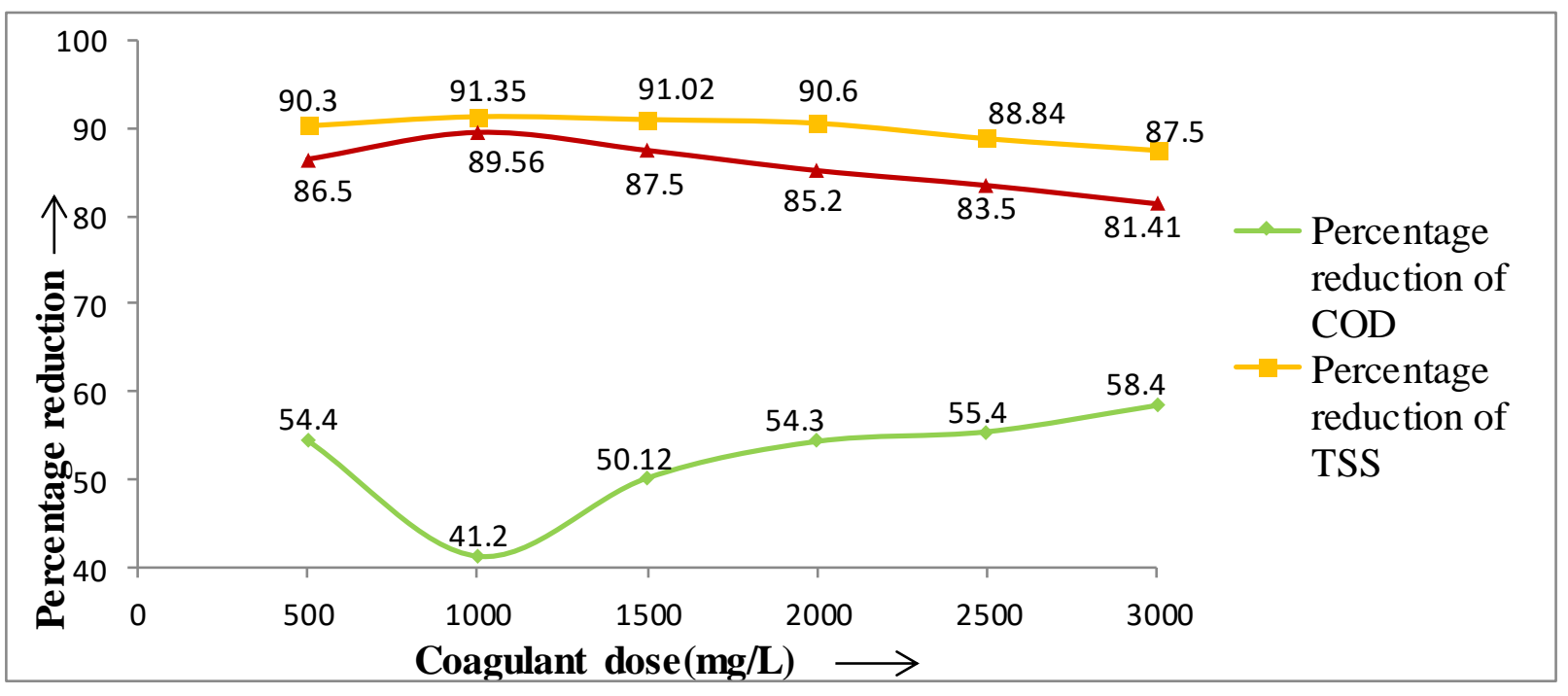

Table 4.2: Optimum dose determination for $\mathrm{ACH}$ Sample: $500 \mathrm{ml}, \mathrm{pH}: 4$

\begin{tabular}{|c|c|c|c|c|c|c|c|c|c|}
\hline \multirow[b]{2}{*}{$\begin{array}{c}\text { Dose } \\
(\mathrm{mg} / \mathrm{L})\end{array}$} & \multicolumn{3}{|c|}{ Initial } & \multicolumn{3}{|c|}{ Final } & \multicolumn{3}{|c|}{ Percent reduction } \\
\hline & $\begin{array}{c}\text { COD } \\
(\mathrm{mg} / \mathrm{L})\end{array}$ & TSS (mg/L) & $\begin{array}{c}\text { Turbidity } \\
\text { (NTU) }\end{array}$ & $\begin{array}{c}\text { COD } \\
(\mathrm{mg} / \mathrm{L})\end{array}$ & $\begin{array}{c}\text { TSS } \\
(\mathrm{mg} / \mathrm{L}) \\
\end{array}$ & $\begin{array}{c}\text { Turbidity } \\
\text { (NTU) }\end{array}$ & COD & TSS & Turbidity \\
\hline 200 & 880 & 990 & 670 & 250 & 235 & 117.6 & 70.48 & 74.5 & 82.44 \\
\hline 400 & 880 & 990 & 670 & 220 & 222 & 103.7 & 72.5 & 76.8 & 84.51 \\
\hline 600 & 880 & 990 & 670 & 225 & 189 & 85.1 & 74.8 & 77.5 & 87.29 \\
\hline 800 & 880 & 990 & 670 & 215 & 160 & 73.3 & 75.46 & 79.5 & 89.05 \\
\hline 1000 & 880 & 990 & 670 & 180 & 194 & 79.8 & 76.8 & 78.2 & 88.08 \\
\hline 1200 & 880 & 990 & 670 & 150 & 210 & 93.2 & 78.9 & 76.8 & 86.08 \\
\hline
\end{tabular}




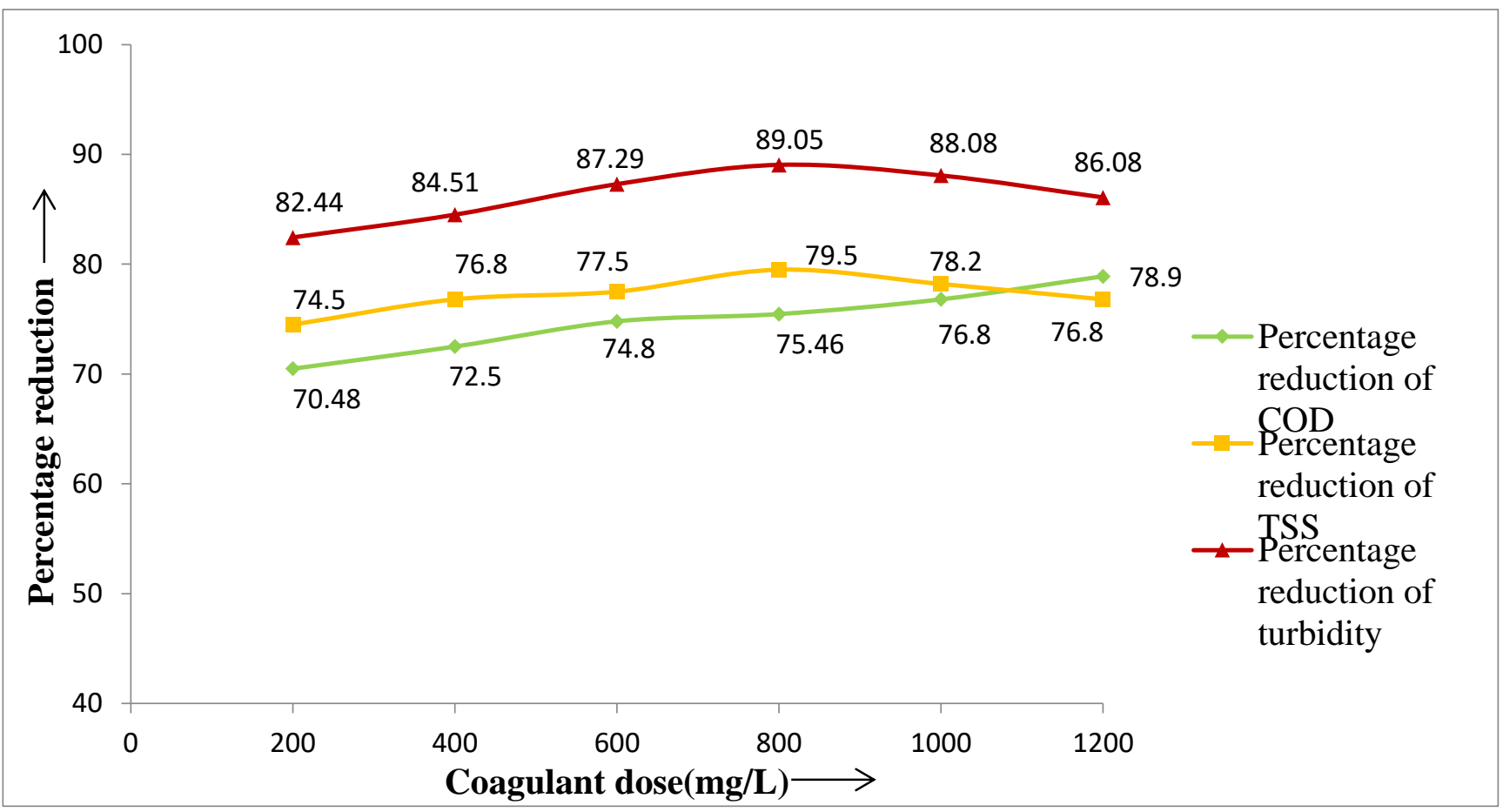

Table 4.3: Optimum dose determination for $\mathrm{MgCl}_{2}$

Sample: $500 \mathrm{ml}, \mathrm{pH}: 4$

\begin{tabular}{|c|c|c|c|c|c|c|c|c|c|}
\hline \multirow[b]{2}{*}{$\begin{array}{c}\text { Dose } \\
(\mathrm{mg} / \mathrm{L})\end{array}$} & \multicolumn{3}{|c|}{ Initial } & \multicolumn{3}{|c|}{ Final } & \multicolumn{3}{|c|}{ Percent reduction } \\
\hline & $\begin{array}{c}\text { COD } \\
(\mathrm{mg} / \mathrm{L})\end{array}$ & $\begin{array}{c}\text { TSS } \\
(\mathrm{mg} / \mathrm{L})\end{array}$ & $\begin{array}{c}\text { Turbidity } \\
\text { (NTU) }\end{array}$ & $\begin{array}{c}\text { COD } \\
(\mathrm{mg} / \mathrm{L})\end{array}$ & TSS (mg/L) & $\begin{array}{l}\text { Turbidity } \\
\text { (NTU) }\end{array}$ & COD & TSS & $\begin{array}{c}\text { Turbidit } \\
\mathrm{y}\end{array}$ \\
\hline 2500 & 845 & 990 & 670 & 375 & 208 & 113.6 & 55 & 70 & 80 \\
\hline 3000 & 845 & 990 & 670 & 340 & 177 & 87.8 & 58 & 75 & 81.5 \\
\hline 3500 & 845 & 990 & 670 & 335 & 157 & 72.9 & 59.8 & 76.4 & 83.5 \\
\hline 4000 & 845 & 990 & 670 & 360 & 191 & 81.3 & 58 & 75.4 & 82.2 \\
\hline 4500 & 845 & 990 & 670 & 320 & 197 & 85.2 & 58.3 & 74.5 & 81.27 \\
\hline 5000 & 845 & 990 & 670 & 315 & 214 & 98.4 & 58.2 & 72 & 82.31 \\
\hline
\end{tabular}

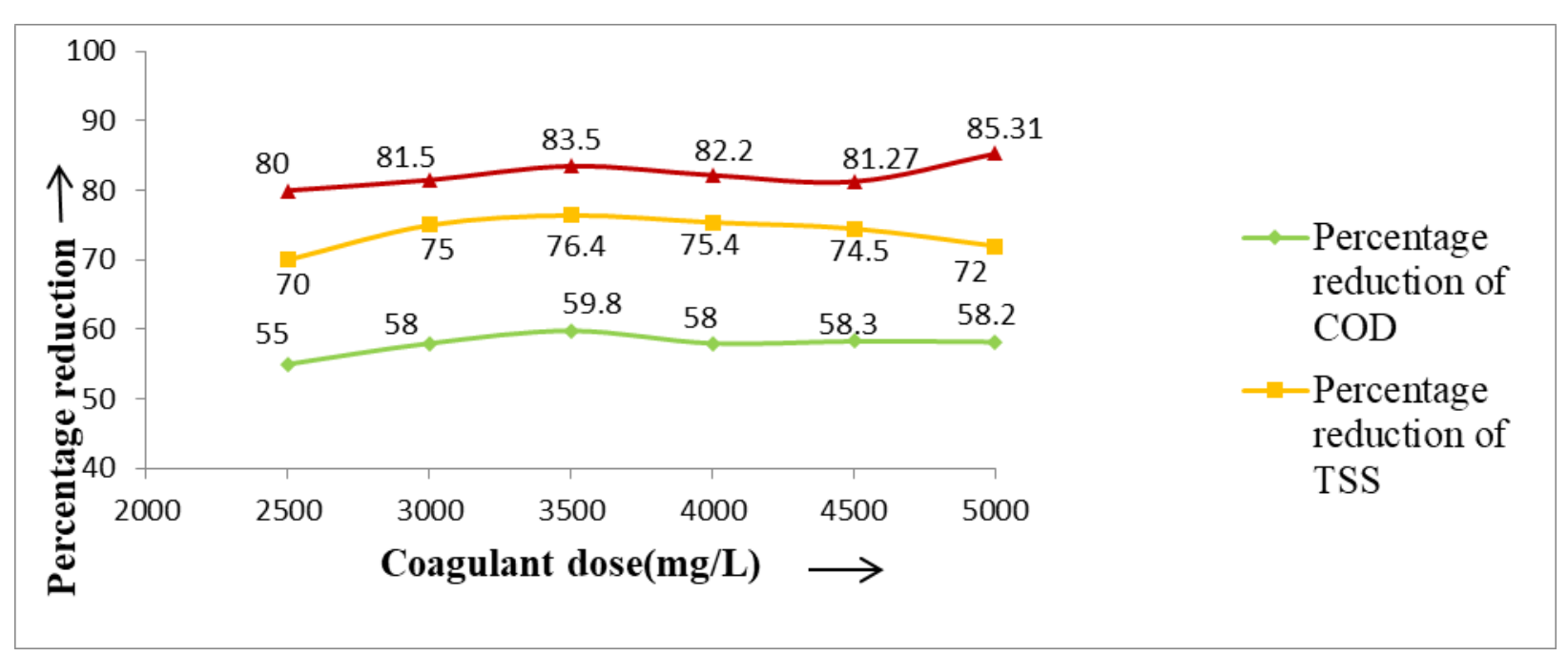


Table 4.4: Optimum dose determination for Poly-Glu Sample: $1000 \mathrm{ml}, \mathrm{pH}: 8$

\begin{tabular}{|c|c|c|c|c|c|c|c|c|c|}
\hline \multirow[b]{2}{*}{$\begin{array}{l}\text { Dose } \\
(\mathrm{ml} / \mathrm{L})\end{array}$} & \multicolumn{3}{|c|}{ Initial } & \multicolumn{3}{|c|}{ Final } & \multicolumn{3}{|c|}{ Percent reduction } \\
\hline & $\begin{array}{c}\text { COD } \\
(\mathrm{mg} / \mathrm{L})\end{array}$ & $\begin{array}{c}\text { TSS } \\
(\mathrm{mg} / \mathrm{L})\end{array}$ & $\begin{array}{c}\text { Turbidity } \\
\text { (NTU) }\end{array}$ & $\begin{array}{c}\text { COD } \\
(\mathrm{mg} / \mathrm{L})\end{array}$ & $\begin{array}{c}\text { TSS } \\
(\mathrm{mg} / \mathrm{L})\end{array}$ & $\begin{array}{c}\text { Turbidity } \\
\text { (NTU) }\end{array}$ & COD & TSS & Turbidity \\
\hline 1 & 795 & 990 & 670 & 280 & 266 & 117.1 & 62.8 & 71.5 & 80.2 \\
\hline 1.2 & 795 & 990 & 670 & 250 & 278 & 134 & 66.4 & 70.5 & 78 \\
\hline 1.4 & 795 & 990 & 670 & 245 & 306 & 142.9 & 67.8 & 68 & 77.5 \\
\hline 1.6 & 795 & 990 & 670 & 240 & 275 & 124 & 67.4 & 70.2 & 80.2 \\
\hline 1.8 & 795 & 990 & 670 & 220 & 205 & 75.6 & 71.5 & 77.5 & 85 \\
\hline 2 & 795 & 990 & 670 & 215 & 214 & 104.9 & 71 & 76.5 & 83.2 \\
\hline
\end{tabular}

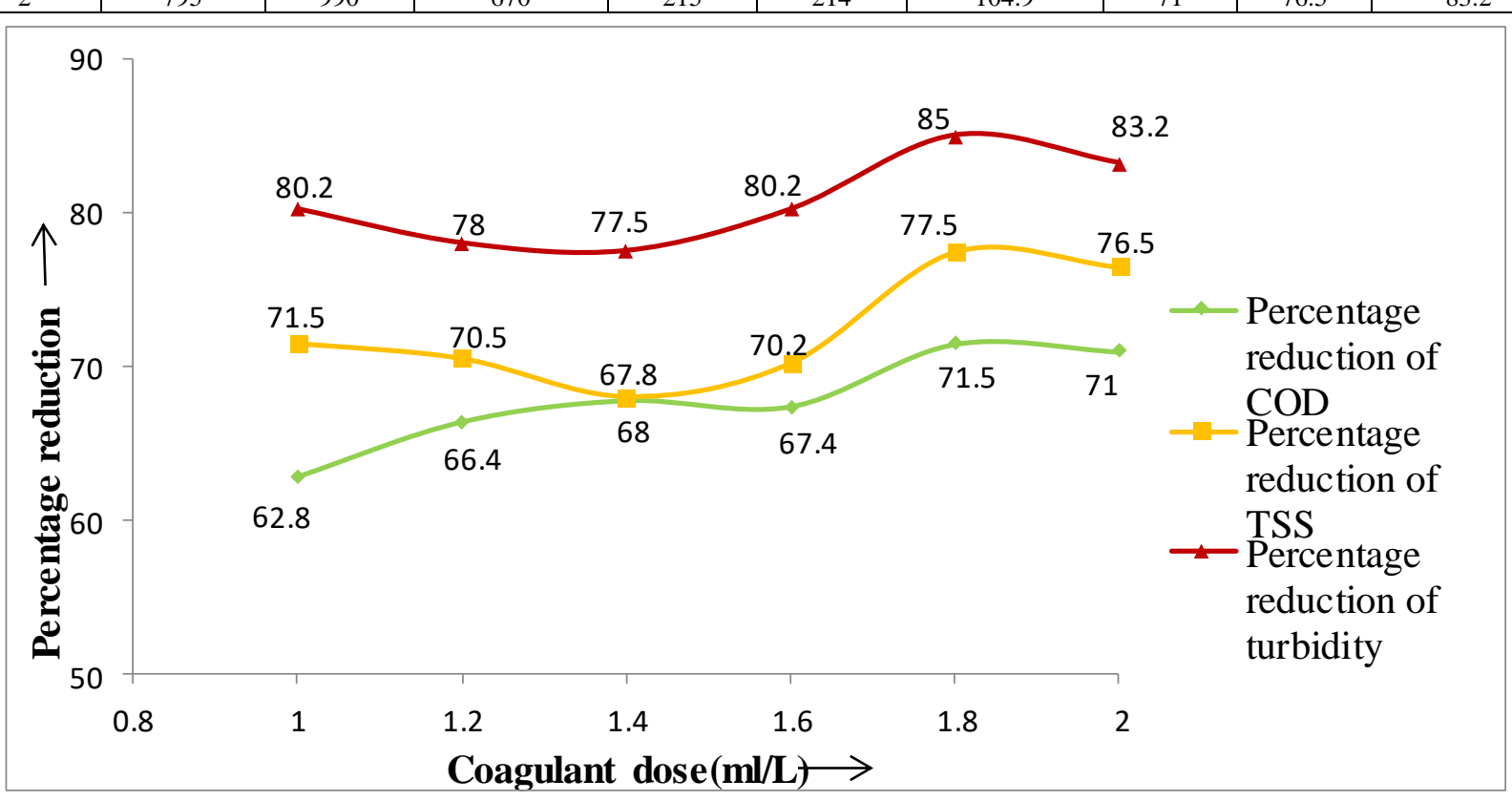

V EFFICIENCY ANALYSIS (H)

Table 5.1: Efficiency for Poly Aluminium Chloride (PAC)

\begin{tabular}{|c|c|c|c|}
\hline Parameter & Weight(A) & Optimum percentage(B) & A x B \\
\hline COD & 30 & 41.2 & 1236 \\
\hline Turbidity & 35 & 91.35 & 3197.25 \\
\hline TSS & 35 & 89.56 & 3134.6 \\
\hline \multicolumn{2}{|c|}{$\eta=\frac{7568}{100}=75.68 \%$} & $\sum 567.85$ \\
\hline \multicolumn{2}{|c|}{}
\end{tabular}

Table 5.2: Efficiency for Aluminium Chlorohydrate (ACH)

\begin{tabular}{|c|c|c|c|}
\hline Parameter & Weight(A) & Optimum percentage(B) & A x B \\
\hline COD & 30 & 75.46 & 2263.8 \\
\hline Turbidity & 35 & 79.5 & 2782.5 \\
\hline TSS & 35 & 89.05 & 3116.75 \\
\hline \multicolumn{2}{|c|}{$\eta=\frac{9168}{100}=81.63 \%$} \\
\hline \multicolumn{2}{|c|}{}
\end{tabular}

Table 5.3: Efficiency for magnesium chloride

\begin{tabular}{|c|c|c|c|}
\hline Parameter & Weight(A) & Optimum percentage(B) & A x B \\
\hline COD & 30 & 59.8 & 2694 \\
\hline Turbidity & 35 & 76.4 & 2922.5 \\
\hline TSS & 35 & 83.5 & $\sum=7390.5$ \\
\hline \multicolumn{2}{|c|}{$\eta=\frac{7291}{100}=73.91 \%$} \\
\hline
\end{tabular}

Table 5.4: Efficiency for Poly-Glu

\begin{tabular}{|c|c|c|c|}
\hline Parameter & Weight(A) & Optimum percentage(B) & A x B \\
\hline COD & 30 & 71.5 & 2145 \\
\hline Turbidity & 35 & 77.5 & 2712.5 \\
\hline TSS & 35 & 85 & 2975 \\
\hline \multicolumn{2}{|c|}{$\eta=\frac{7838}{100}=78.33 \%$} & \\
\hline \multicolumn{2}{|c|}{}
\end{tabular}




\section{CONCLUSIONS}

Summary of the conclusions from the experimental investigations are summarized below.

$>$ It is low cost method for industrial waste water treatment.

$>$ The treatment system is eco-friendly.

$>$ The efficiency of $\mathrm{ACH}$ is good than the other coagulant

$>$ The new era coagulant removed approximate COD $(84 \%)$, BOD $(92 \%)$, TSS $(87 \%)$, and total hardness $(70 \%)$ from the industrial waste water.

$>$ The overall performance of the new era coagulant is excellent than the conventional coagulant

$>$ These treatment technology is alternative to conventional treatment.

\section{REFRENCEC}

[1] A H M Faisal Anwar (2012), "Reuse of laundry grey water in irrigation and its effect on soil hydrologic parameters", International conference on future environment and energy, IPCBEE vol 28 (@012), IACSIT Press, Singapore

[2] A. Khatun \& M.R. Amin, (2011), Greywater reuse: a sustainable solution for water crisis in Dhaka, Bangladesh", 4th Annual Paper Meet and 1st Civil Engineering Congress, Dhaka, Bangladesh ISBN: 978-984-33-4363-5, pp 427-434

[3] Amr M. Abdel-Kader, "Studying the efficiency of grey water treatment by using rotating biological contractors system," Journal of King Saud University Engineering science, May (2012), pp 1-7.[

[4] B. Jefferson, A. Palmer, P. Jeffrey, R. Stuetz and S. Judd, "Grey water charecterisation and its impact on the selection and operation of technologies for Urban reuse", Journal of water science and Technology, Vol. 50, pp 157-164, (2004

[5] Bhausaheb L Pangarkar, Saroj Parjane and M.G. Sane, "Design and Economical performance of Grey water treatment plant in Rural region," International Journal of civil and Environmental Engineering 2:1, 2010

[6] Dr. Mark Pidou, Dr. Fayyaz Ali Memon, Prof. Tom Stepenson, Dr. Bruce Jefferson and Dr. Paul Jefferey, " Grey water recycling:A reviw of Treatment options and applications", Institution of Civil Engineers, proceedings in the journal engineering Sustainability, Vol. 160, pp 119-131

[7] E. Friedler, R. Kovalio and N.I. Galil, "on site grey water treatment and reuse in multi storey buildings," Journal of water science \& Technology Vol. 151, No. 1, pp 187-194. @ IWA Publishing (2005) 